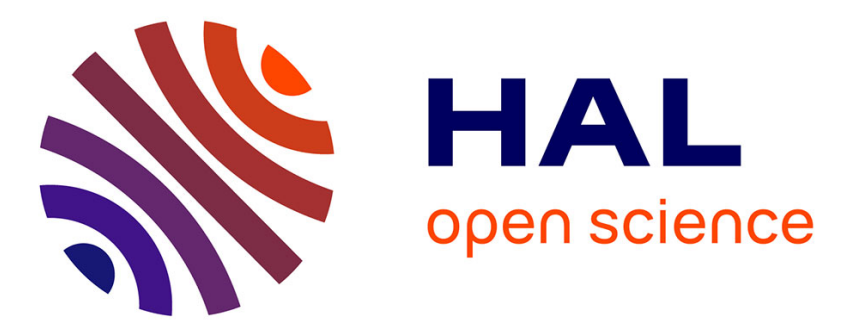

\title{
Layer-by-layer functionalization of gold nanorods by polyelectrolytes to control surface spacer and precisely tune the surface plasmon resonance position
}

\author{
Céline Jégat, Guillaume Laurent
}

\section{- To cite this version:}

Céline Jégat, Guillaume Laurent. Layer-by-layer functionalization of gold nanorods by polyelectrolytes to control surface spacer and precisely tune the surface plasmon resonance position. Chemistry Letters, 2020, 10.1246/cl.200687 . hal-03065980

\section{HAL Id: hal-03065980 \\ https://hal.science/hal-03065980}

Submitted on 23 Nov 2021

HAL is a multi-disciplinary open access archive for the deposit and dissemination of scientific research documents, whether they are published or not. The documents may come from teaching and research institutions in France or abroad, or from public or private research centers.
L'archive ouverte pluridisciplinaire HAL, est destinée au dépôt et à la diffusion de documents scientifiques de niveau recherche, publiés ou non, émanant des établissements d'enseignement et de recherche français ou étrangers, des laboratoires publics ou privés. 


\title{
Layer-by-layer functionalization of gold nanorods by polyelectrolytes to control surface spacer and precisely tune the surface plasmon resonance position
}

\author{
Céline Jégat and Guillaume Laurent* \\ Université Paris-Saclay, ENS Paris-Saclay, CNRS, PPSM, 91190 Gif-sur-Yvette, France
}

E-mail: guillaume.laurent@ens-cachan.fr

We present a layer-by-layer functionalization of gold nanorods (GNRs) by polyelectrolytes in order to control precisely the spacer surrounding the metallic nanoparticles but also to modulate its plasmonic properties. Alternate functionalization with negatively and positively charged polyelectrolytes ensures a $2 \mathrm{~nm}$ step control of the spacer from $0 \mathrm{~nm}$ up to $10 \mathrm{~nm}$, depending on the number of layers. Such process changes the local refractive index and thus induces a red shift of the surface plasmon resonance. Both solution and solid samples were studied.

Keywords: gold nanoparticles functionalization, polyelectrolytes layers, spacer thickness control, plasmonic properties modulation

The enhancement of the metallic nanoparticles synthesis protocols impacts since many years the field of materials science. ${ }^{1-3}$ In fact, controlling precisely the size, shape and the core metal gives access to specific plasmonic properties. Numerous studies describe them and their various applications in designing new meta-materials, sensing and biomedicine $\mathrm{e}^{4-8}$ each underlying that the control of the direct environment of the metallic nanoparticle is mandatory whatever we consider inter-particles coupling or targeted organic compound functionalized on the metallic nanoparticles. ${ }^{9}$ Different methods experienced the change of their initial surfactant coating by other appropriate spacer. Two of the most commonly used are the DNA linker ${ }^{10}$ and the silica shell coating. ${ }^{11}$ The first one shows the advantage of having a precise length for the linker and allows designing either hybrid organic / metallic system or dimer nanoparticles. Despite it is quite easy to use; DNA linker is still expensive and does not allow a good surface coverage in case of organic functionalization. Moreover, the stability of the hybrid system is not optimized. On the other hand, silica shell surrounding metallic nanoparticles increases the stability of the system and exhibits a potentially high surface coverage with further functionalization with organic compound using click chemistry. However, it is hard to control the shell thickness for value bellow $10 \mathrm{~nm}$; which is a huge drawback because the value of the enhanced electromagnetic field for plasmonic nanoparticles decreases as the inverse of the cube distance from the surface, enforcing a spacer thickness smaller than $10 \mathrm{~nm} .^{12}$

Considering the different drawbacks of the previous spacers, using polyelectrolytes could represent a new way in the functionalization of metallic nanoparticles. In fact, as polyelectrolytes are organic polymers where the initial monomer is positively or negatively charged, specific electrostatic interactions afford an easy control of the spacer thickness and at the same time increase the colloidal stability of the system. ${ }^{13-16}$ Moreover, controlling the direct environment of the metallic nanoparticles means controlling the local refractive index and thus enables the modulation of the localized surface plasmon resonance (LSPR). ${ }^{17-19}$

In this paper, negative and positive polyelectrolytes are sequentially used to functionalize GNRs. Using such layerby-layer process ensures a perfect control of the spacer surrounding the metallic nanoparticles, an increase of its colloidal stability and a higher density when deposited on glass substrate. Zetametry measurements are performed to characterize the layer-by-layer deposition and the evolution of the colloidal stability over the process. Extinction spectroscopy is used to show the spectral shift observed over the LSPR bands during the layer-by-layer process.

Gold nanorods with specific aspect ratio were prepared by a modified "seed mediated growth method" using binary surfactant mixtures. ${ }^{20}$ In this synthesis procedure, sodium oleate $(\mathrm{NaOL})$ is combined with $\mathrm{CTAB}$ and thus achieves a greater tunability of GNRs dimensions. This anionic surfactant has a double bond that allows the reduction of the gold salt in the absence of other reducers, in this case ascorbic acid (AA), so a lower molar ratio of gold:AA is used compared to the original method. The mixture of CTAB and NaOL also enables the use of lower concentration of surfactant in the reaction. ${ }^{15}$

The reaction has two steps. First, a small quantity of gold salt is reduced using sodium borohydride as a strong reducing agent in order to create small gold nanospheres called "seeds", which will be used as cores of the gold nanorods. Secondly, a growth solution provides the gold salt and the other reactants for the growth of these seeds with the desired size and shape. These reactions are prepared as follows.

Seed Solution: $5 \mathrm{~mL}$ of $0.5 \mathrm{mM} \mathrm{HAuCl} \mathrm{m}_{4}$ is mixed with $5 \mathrm{~mL}$ of $0.2 \mathrm{M} \mathrm{CTAB}$ solution in a beaker. $0.6 \mathrm{~mL}$ of fresh $\mathrm{NaBH}_{4}$ is diluted to $1 \mathrm{~mL}$ with water and then injected to the $\mathrm{Au}(\mathrm{III})-\mathrm{CTAB}$ solution under vigorous stirring. The solution color changes from yellow to brownish yellow and the stirring is stopped after $2 \mathrm{~min}$. The seed solution is aged at room temperature for (at least) $30 \mathrm{~min}$ before use.

Growth solution: $3.5 \mathrm{~g}$ of CTAB and $0.617 \mathrm{~g}$ of $\mathrm{NaOL}$ are dissolved in $125 \mathrm{~mL}$ of water in a $0.5 \mathrm{~L}$ Erlenmeyer flask. $12 \mathrm{~mL}$ of $\mathrm{AgNO}_{3}$ solution $(4 \mathrm{mM})$ is added. The mixture is kept undisturbed at $30^{\circ} \mathrm{C}$ for $15 \mathrm{~min}$ after which $125 \mathrm{~mL}$ of $1 \mathrm{mM} \mathrm{HAuCl}_{4}$ solution is added. The solution becomes colorless after $90 \mathrm{~min}$ of stirring $(700 \mathrm{rpm})$ and 2 mLof $\mathrm{HCl}(37 \mathrm{wt} . \%$ in water, $12.1 \mathrm{M}$ ) is then introduced to adjust the $\mathrm{pH}$. After another $15 \mathrm{~min}$ of slow stirring (400 $\mathrm{rpm}), 0.625 \mathrm{~mL}$ of $0.064 \mathrm{M} \mathrm{AA}$ is added and the solution is vigorously stirred for $30 \mathrm{~s}$. Finally, $0.2 \mathrm{~mL}$ of the seed solution is injected into the growth solution. The resultant 
1 mixture is stirred for $30 \mathrm{~s}$ and left undisturbed at $30^{\circ} \mathrm{C}$ for 12-15 $\mathrm{h}$ for GNRs growth.

The synthesized GNRs were characterized using extinction spectroscopy and scanning electron microscopy (SEM) (Figure 1). They show two different LSPR bands: the transversal one is located at $525 \mathrm{~nm}$ and the longitudinal 7 one at $881 \mathrm{~nm}$. The spectrum is related to the original 8 synthesized solution, within the GNRs are surrounding by a 9 double layer of surfactant (mainly CTAB) and the solvent 10 which contains an excess of surfactant. The size distribution 11 of the GNRs was measured thanks to the SEM images over 12 one thousand nanoparticles. The average values are $97 \mathrm{~nm}$ 13 for the long axis and $21 \mathrm{~nm}$ for the short one, which gives 14 an aspect ratio around 4.6.
$\mathrm{mL}$ of GNRs solution are centrifugated at $6000 \mathrm{rpm}$ during 20 min (relative centrifugal force $\mathrm{RCF}=3461 \mathrm{~g}$ ) and then the pellet is dispersed in $10 \mathrm{~mL}$ of milliQ water. This cycle is done two more times.

Assuming that naked GNRs are slightly positively charged, the first layer needs to be a negatively charged polyelectrolyte; we choose sodium polystyrene sulfonate (PSS, M = $70000 \mathrm{~g} / \mathrm{mol}$ ), purchased from Sigma-Aldrich and used without further purification. So $10 \mathrm{~mL}$ of naked GNRs solution is mixed with $3 \mathrm{~mL}$ of PSS solution (10 $\mathrm{mg} / \mathrm{mL}$ in water) under $2 \mathrm{~min}$ of gentle stirring. The mixed solution is kept undisturbed for $45 \mathrm{~min}$ to allow the electrostatic interactions between the GNRs and the polyelectrolytes thanks to the natural Brownian motion. To remove the excess of polyelectrolytes, centrifugation is performed at $12000 \mathrm{rpm}$ during $10 \mathrm{~min}(\mathrm{RCF}=15455 \mathrm{~g})$ and the pellet is dispersed in $10 \mathrm{~mL}$ of $\mathrm{NaCl}$ solution $(0.5$ $\mathrm{mM}$ ) to prevent the floculation of the hybrid nanoparticles (gold nanorods with one layer, GNRs-1L). The addition of $\mathrm{NaCl}$ increases the ionic strength of the solution which stabilizes the polyelectrolyte layer in water. Without $\mathrm{NaCl}$, the functionalized GNRs precipitate within few hours.

To set the second polyelectrolyte layer, we choose the poly(diallyldimethylammonium chloride) (PDADMAC, M $<100000 \mathrm{~g} / \mathrm{mol}$ ) which is positively charged. The same process is used for the second layer. Briefly, $10 \mathrm{~mL}$ of GNRs-1L are mixed with $3 \mathrm{~mL}$ of PDADMAC solution (purchased from Sigma-Aldrich and used without further purification or dilution). After 2 min of gentle stirring and $45 \mathrm{~min}$ at rest, the same centrifugation parameters are used. The final pellet is dispersed in $10 \mathrm{~mL}$ of $\mathrm{NaCl}$ solution. The use of $\mathrm{NaCl}$ solution instead of milliQ water is very important to prevent the flocculation of the gold nanorods with two layers of polyelectrolytes (GNRs-2L).

We can continue the layer-by-layer process deposition by alternating between PSS and PDADMAC polyelectrolytes, depending on the main charged surface of the hybrids. Because the centrifugation step is more and more critical as the number of layer increases, the rotation speed (or even the RCF) needs to be adjusted in

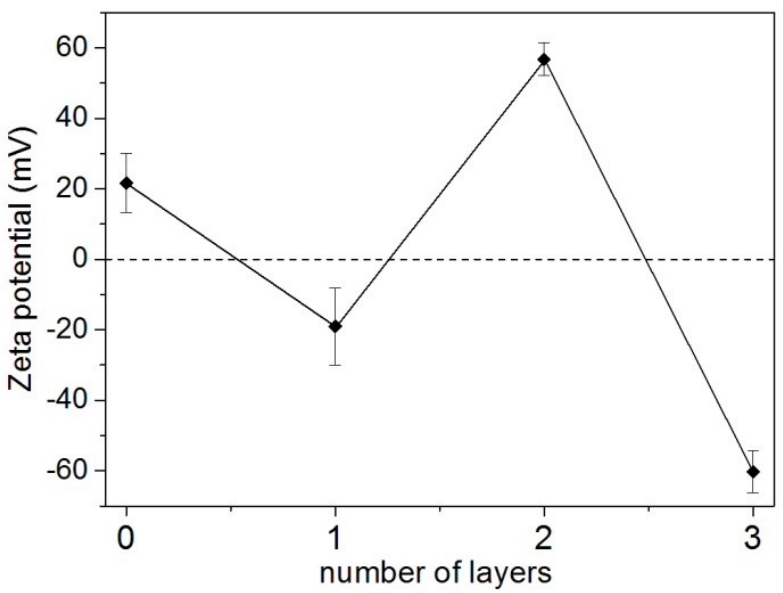

Figure 2. Evolution of the zeta potential of hybrid nanoparticles, starting from naked GNRs (0 layer) to three layers of polyelectrolytes.
Figure 1. a) Extinction spectrum of synthesized gold nanorods. Insert shows the size distribution measured on 1034 nanoparticles; b) SEM image of the corresponding gold nanorods. Scale bar represents $100 \mathrm{~nm}$. 
1 consequence to prevent full aggregation of the pellet (which

2 makes it very difficult to disperse in $\mathrm{NaCl}$ solution).

3 Furthermore, the molecular weight of the different

4 polyelectrolytes were chosen depending on the size (and

5 aspect ratio) of the gold nanorods. If their size increases,

6 longer polyelectrolyte chains are needed.

During the layer-by-layer process, each step was controlled by zetametry. Measuring the GNRs zeta potential, formerly the electrokinetic potential, is an easy way to check if each layer is correctly deposited and at the same time to measure the stability of the hybrid system. Figure 2 shows the variation of the zeta potential when the GNRs are functionalized with up to three layers of polyelectrolytes. First of all, we clearly see the sign alternation of the zeta potential as long as we increase the number of layers. It is positive for the naked GNRs, then negative for the first layer of PSS, positive again for the second layer of PDADMAC and then negative again for the third layer of PSS which is in accordance with the main charge of the last polyelectrolyte deposited. Secondly, as the number of layer increases, the absolute value of the zeta potential increases also, indicating that the stability behavior of the hybrid nanoparticles in solution is more efficient. It starts from 20 $\mathrm{mV}$ for naked GNRs to increase to $60 \mathrm{mV}$ for a three layers system. Considering that each polyelectrolyte layer thickness is around $2 \mathrm{~nm},{ }^{21}$ this layer-by-layer process allows to perfectly controlling the spacer between the surface of the metallic nanoparticle and the future studied organic compound to perform efficient enhanced spectroscopies. This layer-by-layer process is also a good way to prevent aggregation of the gold nanoparticles in solution.

Addition of polyelectrolytes layers around GNRs would also affect their optical plasmonic properties. In fact, LSPR bands are very sensitive to their direct environment, meaning the local refractive index in solution. Thus, putting more layers of polyelectrolytes surrounding the GNRs would change the average value of the refractive index,

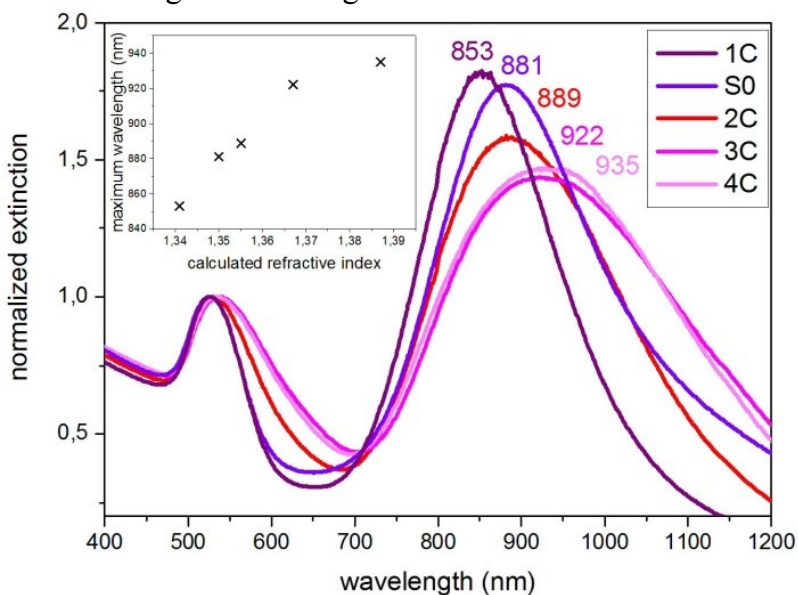

Figure 3. Extinction spectra of functionalized GNRs from 1 to 4 layers of polyelectrolytes. S0 is referred to the synthesized GNRs. Insert shows the evolution of the observed spectral shift with the estimated refractive index.

39 inducing LSPR bands spectral shifts. Figure 3 shows the 40 evolution of the extinction spectra of the GNRs as long as 41 we do the layer-by-layer process. We mainly observe a
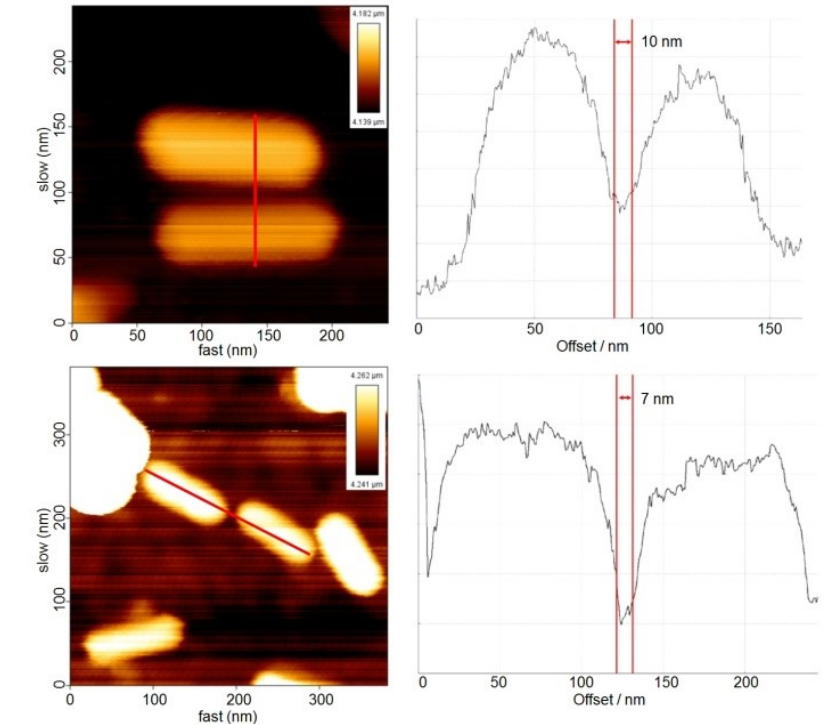

Figure 4. Left: AFM imaging of deposited GNRs-1L. Right: corresponding gap measurements between 2 GNRs-1L. 
1 To go further than these results obtained in solution 2 we considered solid substrates to see how polyelectrolytes 3 can be used to ensure space between GNRs. So we prepared

4 GNRs-1L and deposited a diluted solution by the droplet 5 method on a silicon wafer to perform atomic force 6 microscopy (AFM). Keep in mind that a single PSS layer is

$72 \mathrm{~nm}$ thickness. Figure 4 shows the distance measurement

8 between side-by-side and head-to-head dimers. The average

9 gap value between two GNRs is $8 \mathrm{~nm}$ which clearly 10 indicates that the PSS layer induces repulsive forces 11 between the nanoparticles. It means that polyelectrolytes

12 functionalization prevents the aggregation in solution but 13 also modulates the gap between deposited nanoparticles 14 which is very important when dealing with inter15 nanoparticles' coupling. AFM measurement with more than 16 one layer was not possible due to the quantity of polymer 17 surrounding the GNRs which pollutes the AFM tip and 18 makes imaging complicated. Looking at the study of 19 Möhwald and co-workers ${ }^{21}$, a single layer of polyelectrolyte 20 is $2 \mathrm{~nm}$ thick, two layers are $4 \mathrm{~nm}$ thick and three layers are $216 \mathrm{~nm}$ thick. Considering these layers thickness and the 22 repulsive forces, we can estimate the distance between two 23 GNRs-2L and two GNRs-3L to be over 8 and $12 \mathrm{~nm}$ 24 respectively, when deposited on substrate. deposited on glass slides. Number of polyelectrolyte goes from 0 to 4 layers. samples, it is rather difficult to estimate the value of the local refractive index on deposited sample. In fact, in solution you can consider each GNR individually so only the solvent and the layers have to be considered. When deposited on glass substrate, the direct environment of each GNR is composed of air, glass and other GNRs. So estimating an average value of the local refractive index cannot be efficiently achieved. The second observation is the broadening of both transverse and longitudinal LSPR bands for samples with higher number of layers. This observation is explained by the high density of GNRs that are deposited on the glass substrate. To characterize the GNRs' packing, SEM imaging was performed on GNRs-1L deposited on silicon wafer. Due to vacuum and electron gun source condition, an extra step of oxygen plasma cleaner was done to remove organic compounds like polyelectrolytes and possible rest of surfactant. The main advantage of this extra step is to increase the SEM imaging resolution. But the drawback is that destroying the polyelectrolyte layers changes the distance between the GNRs, especially for high number of layers or when GNRs are closely packed. That is why SEM measurements are only used to characterize the close packing and not the interparticle distances. SEM imaging, in supporting information, clearly show wide areas of high density packing of GNRs. Regarding absorption spectroscopy, these closed packed nanorods induce the broadening of the LSPR bands but also a specific band that appears around $660 \mathrm{~nm}$, due to transverse coupling between GNRs allowed by the short inter-particles distance. ${ }^{23}$ Thus increasing the number of polyelectrolytes layers allows to tune the LSPR bands position but also to modulate the inter-particles coupling by controlling the distance between GNRs.

In conclusion, control of the distance between metallic nanoparticles and targeted organic dye is mandatory for enhanced spectroscopies. Different spacers can be used like silica shell or DNA but polyelectrolytes show many advantages. First of all, as it is a layer-by-layer process, you can control precisely the thickness of the spacer with a $2 \mathrm{~nm}$ single layer precision and also increase the colloidal stability of the functionalized gold nanoparticles. Then, as any modification of the GNRs' surface, putting some electrolytes modulates the position of the LSPR bands, but its linear dependence makes it easily predictable. Finally, for surface deposition, functionalized GNRs can grant access to selection of the gap between nanoparticles which govern the strength of inter-particles coupling and thus, the local field enhancement. To summarize, polyelectrolytes offer new possibilities in term of spacer control and further metallic nanoparticle's functionalization, especially with charged organic compounds.

Co-authors would like to thank François Brisset from ICMMO laboratory, Université Paris-Saclay, France for the scanning electronic microscopy measurements.

Supporting Information is available on




\section{References and Notes}

21 W. L. Barnes, A. Dereux, T. W. Ebbesen, Nature 2003, 424, 824.

2 J. Pérez-Juste, I. Pastoriza-Santos, L. M. Liz-Marzan, P. Mulvaney, Coordination Chemistry Reviews 2005, 249, 1870.

3 C. Louis and O. Pluchery, in Gold nanoparticles for physics, chemistry and biology, ed. by Imperial College Press, 2012.

4 M. Salbini, T. Stomeo, C. Ciraci, R. Fiammengo, V. Mangini, A. Toma, F. Pisano, F. Pisanello, T. Verri, D. R. Smith, M. DE Vittorio, Optical Materials Express 2020, 10, 1264.

$5 \quad$ X. Fu, L. Chen, J. Li, M. Lin, H. You, W. Wang, Biosensors \& Bioelectronics 2012, 34, 227.

$6 \quad$ Z. Zhang, Z. Chen, F. Cheng, Y. Zhang, L. Chen, Biosensors \& Bioelectronics 2017, 89, 932.

7 Z. Zhang, Z. Chen, S. Wang, F. Cheng, L. Chen, ACS Applied Materials \& Interfaces 2015, 7, 27639.

$8 \quad$ Z. Zhang, Z. Chen, C. Qu, L. Chen, Langmuir 2014, 30, 3625.

9 M. Focsan, A. M. Gabudean, A. Vulpoi, S. Astilean, J. Phys. Chem. C 2014, 118, 25190.

10 S. Vial, D. Nykypanchuk, F. L. Deepak, M. Prado, O. Gang, Journal of Colloid and Interface Science 2014, 433, 34.

11 A. Guerrero-Martinez, J. Pérez-Juste, L. M. Liz-Marzan, $A d v$. Mater. 2010, 22, 1182 .

12 P. Reineck, D. Gomez, S. Hock Ng, M. Karg, T. Bell, P. Mulvaney, U. Bach, ACS Nano 2013, 7, 6636.

13 D. I. Gittins, F. Caruso, J. Phys. Chem. B 2001, 105, 6846

14 G. Schneider, G. Decher, Langmuir 2008, 24, 1778.

15 L. L. Rouhana, J. A. Jaber, J. B. Schlenoff, Langmuir 2007, 23, 12799.

16 J. Casas, M. Venkataramasubramani, Y. Wang, L. Tang, Elsevier 2013, 49, 525 .

17 B. N. Khlebtsovabandrey, M. Burovanikolai, G. Khlebtsov, Applied Materials 2019, 15, 67.

18 D. A. Boyne, M. H. Griep, Plasmonics 2018, 13, 1227.

19 F. Kang, J. He, T. Sun, Z. Yong, B. Feng, W. Dang, Y. Lei, Advanced Functional Materials 2017, 27, 1701842.

20 X. Ye, C. Zheng, J. Chen, Y. Gao, C. B. Murray, Nano Letters, 2013, 13, 765 .

21 G. B. Sukhorukov, E. Donath, H. Lichtenfeld, E. Knippel, M. Knippel, A. Budde and H. Möhwald, Colloids Surfaces A: Physiochem Eng. Aspects 1998, 137, 253

22 P. Anger, P. Bharadwaj, L. Novotny, Phys. Rev. Lett. 2006, 96, 113002.

23 M. Funston, C. Novo, T. J. Davis, P. Mulvaney, Nano Letters 2009, 9, 1651. 